\title{
Le syndicalisme agricole
}

\section{François Facchini et Raul Magni-Berto}

\section{(2) OpenEdition \\ Journals}

\section{Édition électronique}

URL : http://journals.openedition.org/economierurale/809

DOI : $10.4000 /$ economierurale.809

ISSN : 2105-2581

\section{Éditeur}

Société Française d'Économie Rurale (SFER)

\section{Édition imprimée}

Date de publication : 10 août 2009

Pagination : 7-13

ISSN : 0013-0559

\section{Référence électronique}

François Facchini et Raul Magni-Berto, «Le syndicalisme agricole », Économie rurale [En ligne], 312 | Juillet-août 2009, mis en ligne le 05 juillet 2011, consulté le 21 décembre 2020. URL : http:// journals.openedition.org/economierurale/809; DOI : https://doi.org/10.4000/economierurale.809 


\section{Introduction \\ LE SYNDICALISME AGRICOLE}

\section{François FACCHINI \\ Université de Reims et CES LAEP Paris I}

\author{
Raul MAGNI-BERTON \\ Spirit CNRS-Science Po de Bordeaux
}

C ette entrée en matière se propose de présenter les raisons du colloque $\mathrm{e}^{(1)}$ dont sont issus les articles de ce numéro spécial d'Economie rurale sur le syndicalisme agricole, de situer les textes publiés dans leur contexte scientifique et de donner aux lecteurs de la revue un certain nombre d'informations sur le travail des économistes qui font l'hypothèse de l'homo oeconomicus pour expliquer l'action collective, l'élaboration des politiques publiques et le vote.

À l'origine de ce colloque il y avait une raison conjoncturelle, les élections professionnelles aux Chambres d'agriculture de 2007 et deux raisons académiques, réunir les travaux des économistes sur la manière dont s'élaborent les politiques agricoles, d'une part, et faire le point sur les travaux des autres disciplines, d'autre part. L'appel à communication indiquait quatre thèmes de réflexion :

- les raisons qui incitent les agriculteurs à adhérer aux syndicats agricoles ${ }^{2}$;

- le financement et les modes de gestion des organisations syndicales du monde agricole ;

- le rôle des syndicats dans la définition et la gestion des politiques agricoles, nationales et européennes ${ }^{3}$;

- le rôle du cadre légal dans les élections professionnelles aux Chambres d'agriculture ${ }^{4}$.

Tous ces thèmes ont déjà fait l'objet d'une importante littérature. Le premier objectif d'une telle manifestation était de s'assurer que la réalité n'était pas venue contredire les modèles théoriques existants. Le deuxième objectif était de renouveler la théorie afin de voir des choses qui n'avaient pas été traitées par les anciennes théories. Les articles publiés dans ce numéro tentent de répondre à ces deux exigences.

1. Colloque organisé par la SFER, en partenariat avec SPIRIT (Science, Politique, Relation internationale, Territoire), les 26 et 27 avril 2007 à l'Institut d'étude politique de Bordeaux.

2. Purseigle F. (2004). Les sillons de l'engagement. Jeunes agriculteurs et action collective. Paris, L'Harmattan INJEP. Le Guen R. (2008). L'engagement professionnel agricole, entre militantisme et services. Revue Pour, $\mathrm{n}^{\circ}$ 196-197, mars, p. 114-130.

3. Tavernier Y. (1962). « Le Syndicalisme paysan et la politique agricole du gouvernement (juin 1958-1962)». Revue Française de Science Politique, vol. 12, n³ 3, p. 599-646. Tavernier Y. (1966). « Le Syndicalisme paysan et la Cinquième République, 1962-1965 ». Revue Française de Science Politique, vol. 16, n 5, p. 869-912. Wilson F. (1983). «Les groupes d'intérêt sous la Cinquième République. Test de trois modèles théoriques de l'interaction entre groupes et gouvernement ». Revue Française de Science Politique, vol. 11, $\mathrm{n}^{\circ} 3$, p. 541-568. Sheingate A.-D. (2000). "Institutions and Interest Group Power: Agricultural Policy in the United States, France, and Japan.” Studies in American Political Development, 14, Fall, p. 184-211. Pesche D. (2000). Le Syndicalisme agricole spécialisé en France. Paris, L'Harmattan. Sheingate A.-D. (2001). The Rises of the Agricultural Welfare State: Institutions and Interest Group Power in the United States, France, and Japan, Princeton, NJ: Princeton University Press. Skogsatd G. (2002). "The Rises of the Agricultural Welfare State: Institutions and Interest Group Power in the United States, France, and Japan”. The American Political Science Review, vol. $96, \mathrm{n}^{\circ} 1$, p. 248-249.

4. Duboscq P., Baratra M., Menault J. et Peronnet M. (1987, p. 3). « 1983, élections aux Chambres départementales d'agriculture : mythe et vecteur ». Association française de science politique, colloque "Les agriculteurs et la politique depuis 1970 » Paris. Magimel J. (1995). «Élections aux Chambres d'agriculture. Les leçons d'un scrutin ». Revue Paysans, n² 230, p. 5-17. Le Gen R. (1995). «Les élections aux Chambres départementales d'agriculture du 31 janvier 1995 : un révélateur du changement d'identité professionnelle des agriculteurs ». Économie rurale, 237, p. 30-36. Minot D. (1997). « Les élections aux Chambres d'agriculture de 1983 ». Économie rurale, 237, p. 27-29. 
Le colloque a été ouvert par trois interventions de nature générale de Pierre Muller, Ali Aït Abdelmalek et Jean Vercherand ${ }^{5}$. Ils ont chacun à leur manière défini les enjeux de la recherche sur le syndicalisme agricole pour les sciences politiques, la sociologie et la socioéconomie. Sur les dix-huit communications présentées à l'Institut d'étude politique (IEP) de Bordeaux, sept étaient le fait de sociologue, sept de politiste, trois d'économiste et une d'un historien. Le faible nombre des historiens ne traduisait pas le désintérêt de ces derniers pour le sujet, mais des problèmes de tradition et de financement de la recherche. L'histoire a d'ailleurs été présente tout en long des débats, la plupart des travaux exposés mobilisant les travaux des historiens (Barral, 1968 ; Boussard, 1980 ; Prugnaud, 1963 ; Bruneteau, 1994 ; Martin 2005) ${ }^{6}$.

L'étude du syndicalisme agricole reste très sensible au jeu de complicité et de concurrence (Maresca, 1989)7 qui existe toujours aujourd'hui entre l'intérêt des savants et l'intérêt politique sur la question du syndicalisme agricole. On peut d'ailleurs étendre ce constat à l'ensemble des travaux sur la politique agricole. Dans ce domaine les savants sont plutôt des politistes et des sociologues. Ces disciplines ont, notamment, décrit avec précision le système de cogestion que les organisations professionnelles agricoles ont réussi à mettre en place avec l'accord des gouvernements durant toute la seconde moitié du $\mathrm{XX}^{\mathrm{e}}$ siècle ${ }^{8}$. Les colloques réguliers de l'Association française de sciences politiques (AFSP) sur l'agriculture et la politique ont, sur ce thème, été des moments importants pour faire une sorte de bilan régulier des connaissances accumulées 9 .

Cependant, cette importance des travaux effectués par les sciences politiques et la sociologie ne doit pas nous faire ignorer les contributions de la théorie des choix publics et de la figure de l'homo oeconomicus aux différents thèmes du colloque :

- l'engagement des individus dans l'action collective ;

- le montant des budgets mobilisés ;

- les décisions publiques en matière de politique agricole ;

- les déterminants économiques du vote.

5. Muller P. (1984). Le technocrate et le paysan. Édition ouvrières, Paris. Ait Abdelmalek Ali (1993). Entre l'Europe communautaire et l'exploitation familiale, le rôle des médiations institutionnelles et organisationnelles. L'exemple du Pays de Redon. Paris, thèse de Doctorat (nouveau régime) de l'EHESS, sous la direction de Dominique Schnapper, directeur d'études à l'EHESS, 622 p., publié sous forme abrégée, (1996), collection Alternatives rurales, Paris, Éditions L'Harmattan, 316 p. Vercherand J. (1994). Un siècle de syndicalisme agricole : la vie locale et nationale à travers le cas du département de la Loire. Publications de l'Université de Saint-Étienne.

6. Barral P. (1968). Les Agrariens français de Méline à Pisani. Paris, Ed. Colin, Cahiers FNSP n ${ }^{\circ} 164$. Boussard I. (1980). Vichy et la corporation paysanne. Presses de la FNSP. Bruneteau B. (1994). Les paysans dans l'État : le gaullisme et le syndicalisme agricole sous la Vீ République. Paris, L'Harmattan, Coll. L'alternative rurale. Prugnaud (1963). Les étapes du syndicalisme agricole en France. Paris, Ed. de l'EPI. Martin J.-P. (2005). Histoire de la nouvelle gauche paysanne. Des contestations des années 1960 à la Confédération paysanne. Paris, La Découverte.

7. Maresca S. (1989). «Intérêts Savants et intérêts politiques. Les intellectuels français et la question du syndicalisme agricole ». Recherches Sociologiques, vol. 20, n 3, p. 383-402.

8. Cordelier S. (1990). « La gauche paysanne moderne et la cogestion». In P. Coulomb et alii « Les agriculteurs et la politique », Paris, Presses de Sciences Po, Coulomb P. (1990). « La cogestion, une nouvelle tentative corporatiste » et «Les conférences annuelles entre corporatisme et populisme ». In P. Coulomb et alii «Les agriculteurs et la politique, Paris, Presses de Sciences Po, Roman C. (1990). Agriculture : le système français. Origines et fonctions des organisations professionnelles agricoles. Association pour la Formation et l'information paysanne, Paris, Syros Alternatives. Colson F. (2008). « Que reste-t-il de la cogestion Étatprofession ?». Revue Pour, n 196-197, p. 107-113.

9. Coulomb P., Delorme H., Hervieu B., Jollivet M. et Lacombe P. (dir.) (1987). Les agriculteurs et la politique. Colloque « Les agriculteurs et la politique depuis $1970 »$, Paris. 
Raisonner en termes économiques permet, entre autres, de ne pas oublier que toutes décisions à un coût d'opportunité. Choisir $\mathrm{A}$ sacrifie les autres choix possibles $(\mathrm{B}, \mathrm{C}, \mathrm{D}$, etc.). Lorsqu'un syndicalisme manifeste ou cotise à la vie de son syndicat, il n'investit pas dans son exploitation, ne consomme pas, ne laboure pas et/ou délaisse son épouse. Il sacrifie des ressources (moyen) en vue d'atteindre un objectif. C'est, sur cette base, que la théorie des choix rationnels explique les décisions humaines et, in fine, l'existence des syndicats et de leurs décisions. Elle rappelle que même l'action collective est explicable par des fins individuelles et la mise en œuvre de moyens appropriés pour les atteindre.

\section{L'engagement des individus dans l'action collective}

Dans tous les pays d'Europe les syndicats agricoles ont un taux d'adhérents bien plus important que les syndicats des travailleurs ${ }^{10}$ (tableau 1). Cette forte mobilisation est d'autant plus intéressante qu'il existe des traits spécifiques de la culture française qui conduisent les individus à s'engager moins que d'autres peuples dans l'action collective organisée ${ }^{11}$. La théorie des choix rationnels va expliquer ce fait par les contraintes économiques qui pèsent sur les agriculteurs. Il sera généralement moins coûteux et plus bénéfique pour eux d'agir sur les règles du jeu (la loi) que de chercher une solution aux problèmes qu'ils rencontrent dans le cadre du jeu du libre marché. Cela s'explique notamment par les caractéristiques de leur activité. L'activité agricole est territorialisée. La terre est un facteur de production immobile. Contrairement au capital financier, l'agriculteur ne peut pas faire défection il est obligé de produire sur place. Il va alors être incité à agir sur la collectivité pour valoriser son capital sur place ${ }^{12}$. L'immobilité de leur capital les rend très dépendants des décisions politiques. Ils ont intérêt à contrôler cette décision, à faire pression et même à être élu afin d'agir directement sur les choix politiques.

Tableau 1. Taux de mobilisation des agriculteurs et des travailleurs

\begin{tabular}{c|c|c}
\hline & Syndicats agricoles & Syndicats ouvriers \\
\hline France & 54,5 & 23,0 \\
\hline Allemagne & 90,0 & 40,0 \\
\hline Grande-Bretagne & 78,0 & 50,0 \\
\hline Italie & 80,0 & 36,5 \\
\hline \multicolumn{2}{|r}{} & Source $:$ Keeler (1996)
\end{tabular}

\section{Le montant des budgets}

L'importance du montant des budgets mobilisés par les syndicats dans les choix politiques est généralement sous-estimée. La théorie économique donne, au contraire, une place à l'argent des syndicats dans les choix publics parce qu'elle pense la politique comme un lieu d'investissement. Les groupes d'intérêt investissent en politique comme une entreprise investit dans les nouvelles technologies pour accroître ses profits. Comme il y a des

10. P. Coulomb et al. (1987), cf. la note 4. Voir aussi Keeler JTS (1996). “Agricultural Power in the European Community: Explaining the Fate of CAP and GATT Negociations”. Comparative Politics, 28 (1), p. 127-149. 11. Magni-Berton R. (2009). « Participation politique en France : participer sans militer » In D. Boy, B. Cautrès et N. Sauger «Les français, des européens comme les autres? Une sociologie des opinions européennes », Paris, Les presses de Science Po, à paraître.

12. Nello S.-S. (1984). "An Application of Public Choice Theory to the question of CAP reform”. European Review of Agricultural Eco., 11, p. 261-283. Facchini F. (2006). «L'entrepreneur politique et son territoire », Revue d'Économie Régionale et Urbaine, $\mathrm{n}^{\circ} 2$, p. 263-280. Disponible à l'adresse : cat.inist.fr

13. $C f$. la note 10 . 
stratégies marketing, il y a des techniques de pression. Les syndicats sont donc traités comme des groupes d'intérêt qui s'organisent en groupe de pression pour protéger leurs avantages concurrentiels. La théorie économique rejoint en ce sens un certain nombre de travaux de sciences politiques ${ }^{14}$. Les groupes de pression peuvent réaliser leurs objectifs :

- en agissant sur l'opinion publique pour justifier auprès des élus les transferts d'une partie des impôts vers le secteur agricole ;

- en soutenant politiquement un parti politique ou un candidat (contribution électorale, consigne de vote) ;

- en rencontrant les élus directement afin de les convaincre du bien fondé de leurs revendications ;

- en utilisant la force (manifestation de rue, violence paysanne) ${ }^{15}$.

Il n'est pas improbable, alors, que les groupes d'intérêt qui obtiennent le plus d'aides de l'État soient ceux qui investissent le plus de ressources dans l'activité de pression autrement dit ceux qui utilisent toute la palette de ces techniques de pression. Le financement du syndicalisme agricole français reste cependant encore mal connu. Des travaux comparatifs sur les modes de financement des syndicats agricoles européens et leurs conséquences seraient utiles et devraient être financés. Un financement quasi-public de l'action syndicale ne peut que modifier à terme les stratégies des syndicats et créer des barrières à l'entrée dans l'offre syndicale si certains syndicats ont plus accès aux financements publics que d'autres.

\section{Le rôle des syndicats dans les décisions publiques}

Du financement des syndicats à l'étude de leur rôle dans la formation des politiques publiques il n'y a qu'un pas. C'est sans doute dans ce champ que l'on trouve le plus de travaux ${ }^{16}$. Il s'agit de préciser le rôle des groupes d'intérêts agricoles dans l'élaboration et la mise en œuvre des politiques agricoles. Le montant des aides à l'agriculture et les modalités d'intervention de la politique agricole vont dépendre du montant des investissements politiques des groupes agricoles, de l'intérêt des élus (poids électoral des agriculteurs), du montant des dépenses engagées par les groupes anti-dépenses agricoles ou pro libre échange lors des négociations à l'Organisation mondiale du commerce (OMC), des intérêts de l'Administration publique, de la représentation du monde agricole à l'Assemblée nationale et de la possibilité pour ces élus pro-politiques agricoles d'échanger un vote favorable aux aides agricoles contre un vote pro-politique urbain par exemple.

14. Bonnet A. (1971). Le Syndicalisme agricole en tant que groupe de pression. Paris, Thèse, Science Politique.

15. Mueller D.-C. (2003). "Interest groups, campaign contributions, and lobbying". Public Choice, Cambridge University Press, Chapter 20.

16. Cette note donne quelques-unes de ces références. C. Pondaven (1989). La théorie de la réglementation. Efficacité économique ou efficacité politique ? Application économétrique à la politique agricole commune, LGDJ, Paris. Mayer H.-W., Josling T.-E. (1990). Agricultural Policy Reform - Politics and Process in the EC and the USA, Harvester Wheatsheap, New York, London, Toronto, Sydney, Tokyo. Josling T. (1994). « La réforme de la PAC et son importance pour les pays industrialisés ", Économie Rurale, n 223, p. 27-31. RoedererRynning C. (2006). Farm Conservatism in France: Revisiting the Weak State Thesis. Department of Political Science and Public Management, University of Southern Denmark, Campusvej. Nedergaard P. (2006). "Market failures and government failures: A Theoritical model of the common agricultural policy", Public Choice, 127, p. 393- 413. Swinnen J., Van der Zee F. (1993). "The political economy of agricultural policies: a survey", European Review of Agricultural Economics, 20, p. 261-290. 
Généralement la théorie économique explique la faiblesse des groupes anti-dépensiers face aux groupes agricoles par leur taille ${ }^{17}$ et la répartition des bénéfices et des coûts de la politique agricole ${ }^{18}$. Pour obtenir satisfaction, un groupe ne doit être ni trop grand ni trop petit. S'il est trop grand les bénéfices que chacun retire de l'action collective sont infimes, personne n'a alors intérêt à s'engager. S'il est trop petit les coûts supportés par chacun sont trop élevés. L'exode rural a permis de réduire la taille du groupe des agriculteurs et de baisser ainsi les coûts de son organisation et d'augmenter les bénéfices que chacun peut attendre de l'action collective et des dépenses de pression sur le gouvernement. Inversement les contribuables restent inorganisés car incapables de trouver suffisamment d'intérêt dans une action collective qui aurait pour objectif de réduire les dépenses publiques en faveur des agriculteurs. Les gains seraient d'autant plus faibles qu'ils sont nombreux et que la part des dépenses alimentaires dans leur budget total a eu tendance à baisser au cours du $\mathrm{xx}^{\mathrm{e}}$ siècle. La théorie de l'action collective de Mancur Olson ${ }^{19}$ donne une autre explication. Elle rappelle qu'un groupe d'intérêt ne réussira à imposer sa demande auprès des hommes politiques qui si les transferts publics qu'elle attend ont des coûts diffus (ensemble de la collectivité nationale) et des bénéfices concentrés (profession agricole). En obtenant une telle répartition des coûts et des bénéfices, la profession agricole se couvre contre l'action des groupes anti-dépensiers.

\section{Les déterminants économiques du vote}

L'économie du vote est aussi un domaine où l'hypothèse de l'homo oeconomicus est utilisée pour expliquer, d'une part, la participation à une élection et, d'autre part, l'orientation du vote. L'économie du vote est née avec l'ouvrage de Downs de $1954^{20}$ et l'assimilation qu'il fait de l'homo politicus à un homo oeconomicus. Cette assimilation reste un enjeu pour les sciences sociales. Tout en restant critique, pour un certain nombre d'entre eux, les économistes acceptent l'homo oeconomicus comme une hypothèse de travail. Les sciences politiques et la sociologie y sont, en revanche, plus hostiles (Udehn, 1997 ; Chanial, 1997 ; Merle, 1990 ; Blerald, 1991 ; Green et Shapiro, 1995 ; Lehingue, 1997, 1998) ${ }^{21}$. Malgré l'importance de ses critiques, il est indéniable que les choix électoraux sont influencés par les variables économiques. L'histoire de cette littérature pourrait être écrite en trois vagues. La première vague innove, la seconde multiplie les champs d'application et propose des modèles d'économie du vote complet

17. Pour une application au cas des États-Unis Gardner B.-L. (1992). "Causes of U.S. Farm commodity programs". Journal of Economic Literature, 30, p. 62-101.

18. Pour une application au cas des États-Unis Gardner B.-L. (1992). "Causes of U.S. Farm commodity programs". Journal of Economic Literature, 30, p. 62-101.

19. Olson M. (1965). The Logic of Collective Action. Cambridge, Mass. Harvard University Press. (1985), Théorie de l'action collective. Paris, PUF, coll. Sociologie.

20. Pour une application à la situation française, voir Padioleau G. (1982). L'état au concret. Paris, PUF, coll. Sociologie.

21. Udehn Lars (1997). The Limits of Public Choice. A sociological critique of the economic theory of politics, Routledge, London and New York. Chanial P. (1997). «L'homo politicus. Les paradoxes de la théorie de l'acteur rationnel ». Recherches, 10. Merle P. (1990). «L'homo politicus est-il un homo oeconomicus ? », Revue française de science politique, 40, 1. Blerald P.-A. (1991). « Théorie du marché politique et rationalité des politiques publiques ». Revue Française de Science Politique, 41, n 2, p. 235-263. Green D.-P. et Shapiro, I. (1995). « Choix rationnels et politique. Pourquoi en savons-nous toujours aussi peu? ». Revue Française de Science Politique, vol, $n^{\circ}$ 1, février, p. 96-130. Lehingue P. (1998 et 1997). «L'analyse économique des choix électoraux ou comment choisir d'économiser l'analyse ». Politix, nº 40 et 41, p. 88-112 et p. 82-122. 
comme l'école de Zurich, la troisième cherche à interpréter les résultats empiriques parfois décevants. Elle focalise son attention sur le rôle de l'information dans les choix électoraux (Nannestad et Paldam, 1994)22.

C'est dans ce contexte scientifique général que ce colloque s'est déroulé et que ce numéro est publié. Les articles retenus n'ont, nous semble-t-il, pas l'ambition de proposer une nouvelle théorie dans chacun des domaines qui sont les leurs. Néanmoins, ils répondent tous aux deux exigences citées précédemment. Ils suivent un évènement et l'interprètent à la lumière des faits et des grilles d'interprétation antérieures ou ils proposent d'appliquer des hypothèses théoriques connues à des domaines où jusqu'à présent elles ne les avaient pas été. Deux thèmes de réflexion ont été privilégiés lors de la constitution de ce numéro : - la mobilisation des agriculteurs dans le cadre des Chambres d'agriculture ;

- et les stratégies des syndicats face aux pouvoirs publics.

Les trois premiers articles traitent de l'intérêt des agriculteurs pour l'action collective, les deux suivants des stratégies des syndicats face aux pouvoirs publics.

- Le premier article est écrit par deux sociologues, Serge Cordellier et Roger Le Guen. Il analyse l'évolution récente du syndicalisme agricole à partir d'une interprétation des résultats des élections aux Chambres d'agriculture de 2007. Il constate que l'une des caractéristiques de cette élection est le retour aux urnes des agriculteurs. Il explique cette baisse de l'abstention par la présence d'un réel clivage entre les positions du pôle FNSEACNJA d'une part et la Confédération paysanne, d'autre part. Cette explication prolonge l'un des résultats de l'économie du vote et son hypothèse de l'abstention par indifférence ${ }^{23}$. L'abstention par indifférence est une situation où l'électeur ne perçoit pas de différences significatives entre les positions des différents candidats et les partis en compétition qui justifient la participation. Le clivage entre les deux syndicats réduit ce type d'abstention et explique la bonne participation de 2007.

- Quatre économistes, Eric Dubois, François Facchini, Martial Foucault et Abel François, ont collaboré pour le deuxième article. Il ne traite pas des élections de 2007 pour des raisons de disponibilité des données, mais des déterminants économiques des choix électoraux des agriculteurs des élections professionnelles de 1995 et 2001. Il se différencie de l'article des sociologues par sa méthode et ses hypothèses théoriques. Il étend la théorie économique du vote à un domaine où elle n'a jamais été appliquée. Il ouvre à cette occasion un champ important de recherche pour les économistes du vote, les élections professionnelles (ouvriers, cadres, fonction publique).

- Xabier Itçaina propose le troisième article. Il applique au cas basque une analyse portant sur les initiatives concurrentes et non institutionnelles de remobilisation des agriculteurs. Il met en évidence la façon dont les dynamiques territoriales et identitaires viennent concurrencer les structures catégorielles. Il est utile ici de replacer cette analyse dans le cadre de la politique agricole commune. La dimension régionale des financements de

22. Nannestad P. et Paldam M. (1994). "The VP-function: a survey of the literature on vote and popularity functions after 25 yaers”. Public Choice, 79, p. 213-245.

23. François A., Fauvelle-Aymar C. (2006). "The impact of closeness on turnout: An unambiguous empirical relation based on a study of a two rounds ballot". Public Choice, vol. 127, n 3-4. Voir aussi Kirchgässner G. (2003). "Abstention Because of indifference and Alienation, and Its Consequences for Party Competition: A Simple Psychological Model”. Working Paper Series, CESifo, U of St Gallen Economics Discussion Paper n 2003-12. 
l'Union européenne - associée à la moindre cohésion catégorielle des agriculteurs français - stimule les initiatives identitaires liées au territoire. Même dans un État comme la France où les identités territoriales entraînent traditionnellement une mobilisation relativement faible.

- L'étude des origines, d'une part, et de la mise en forme des modes de représentation des intérêts des agriculteurs en France, d'autre part, fait l'objet du quatrième article. Le sociologue Denis Pesche rappelle l'histoire de l'Association générale des producteurs de blé (AGPB) et autres céréales afin d'expliquer le rôle joué par ce syndicat dans le développement économique du secteur des céréales et le rôle qu'il a joué et qu'il joue encore dans la définition des politiques agricoles. Il définit le syndicalisme agricole comme un produit social et insiste sur l'importance de la constitution de représentations communes dans la formation du groupe des céréaliers. L'idéal du militant de l'AGPB c'est d'être un entrepreneur qui accepte les lois du marché et, à ce titre, participe au développement agricole de la France. Ce que montre Denis Pesche, cependant, c'est l'écart entre l'idéal et la réalité. Car malgré cet appel à la culture entrepreneuriale, les stratégies de l'AGPB restent centrées sur la recherche de marchés contraints, d'aides publiques.

- Enfin, le cinquième et dernier article est le fait de deux économistes. Valérie Bernardi et Jean-Pierre Boinon analysent, dans un cadre plutôt institutionnaliste, le modèle de cogestion au niveau départemental. Cela montre que les syndicats n'agissent pas seulement au niveau international (OMC, Union européenne) et national (État central) mais aussi au niveau des Chambres d'agriculture, des départements et des Régions. Cela permet également de ne pas oublier que les syndicats ne font pas que participer à l'élaboration de la politique agricole. Ils souhaitent aussi agir sur sa mise en œuvre. On trouve là une bonne illustration de l'idée d'Olson selon laquelle les militants, pour participer à la production de biens collectifs, produisent en marge de ces biens, des biens de club, autrement dit des biens qui ne sont accessibles qu'aux proches des syndicats. L'étude montre précisément le rôle des syndicats dans la politique d'installation de six départements français (Somme, Aveyron, Morbihan, Eure, Finistère, Haute-Vienne).

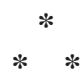

Nous espérons que ce numéro contribuera à une actualisation de nos connaissances sur le syndicalisme agricole et à la mise en place de nouvelles pistes de recherche. Sa composition montre déjà la diversité des méthodes et la richesse des analyses du syndicalisme agricole en science sociale. Le défi était de faire coexister des écoles de pensée et des méthodes qui généralement s'ignorent du fait de la constitution de lignes éditoriales qui rendent de plus en plus étanches les manières de voir les mondes en présence.

Pour cette raison, nous remercions les éditeurs de la revue d'avoir accepté la mise en évidence du pluralisme des approches sans nuire, nous l'espérons, à la qualité des contributions publiées. 